\title{
Modelo conceptual para gestionar la innovación en las empresas del sector servicios
}

\section{Resumen}

\author{
Arzola, Minerva* \\ Mejías, Agustín ${ }^{\star *}$
}

Este artículo tiene por objetivo proponer un modelo conceptual para la gestión y evaluación de la innovación en las empresas del sector servicios. La metodología utilizada para desarrollar esta investigación fue en primer lugar la revisión bibliográfica, luego se analizaron y evaluaron las teorías relacionadas, luego de contrastar esta información se desarrolló un modelo conceptual para gestionar la innovación en las empresas de servicios. El modelo propuesto se fundamenta en los modelos de excelencia de gestión y los utilizados para gestionar y medir la innovación en las empresas; consiste en valorar siete dimensiones, 7D's, en las empresas de servicios, a saber: Liderazgo, Planificación estratégica, Satisfacción de Clientes, Procesos, Organización, Competencias del Recurso Humano y Responsabilidad Social, evaluadas en dos escalas, una del 1 al 5 y la otra por \% de cumplimiento. Como conclusiones de este articulo se tiene que el modelo conceptual propuesto constituye un aporte teórico para la gestión de la innovación en el sector servicios, el cual considera los aspectos administrativos, operativos y de resultados, indispensables para la competitividad de las empresas del sector servicios; y las siete dimensiones propuestas por el modelo 7D's deben interactuar simultáneamente para alcanzar la innovación del servicio esperada por los clientes.

Palabras clave: Sector servicios, modelo conceptual, medición de la innovación, innovación, excelencia de gestión.

\section{A Conceptual Model for Innovative Management in Service Sector Companies}

\section{Abstract}

The purpose of this article is to propose a conceptual model for the management and evaluation of innovation in service sector companies. The methodology used to develop this research was initially bibliographical review, and after analyzing and evaluating the related theories available, it was

\section{Recibido: 09-05-06. Aceptado: 17-11-06}

* Dra. Ingeniero Industrial. Prof. Asociado-Investigadora. UNEXPO-Vicerrectorado Puerto Ordaz. Centro para el Desarrollo Gerencial. E-mail: minervarzola@yahoo.com

** M. Sc. Ingeniero Industrial. Prof. Agregado-Investigador. Universidad de Carabobo. Grupo de Investigación en Gestión de la Calidad. E-mail: amejiasa@uc.edu.ve 
contrasted and a conceptual model to manage innovation in service enterprises was developed. The proposed model is based on management excellence models and used to manage and to measure innovation in companies, and consists of evaluating seven dimensions, 7D's, in service companies, namely: leadership, strategic planning, customer satisfaction, processes, organization, human resources competence, and social responsibility, evaluated on two scales, one from 1 to 5 and the other by percentage of fulfillment. As conclusions this paper asserts that the proposed conceptual model constitutes a special contribution to innovation management companies in the service sector, which considers administrative, operative and end product results. It is indispensable for competitiveness of companies in the service sector; and the seven proposed dimensions of the model 7D must interact simultaneously in order to enhance innovation in the services expected by clients.

Key words: Service sector, conceptual model, innovation measurement, innovation, business excellence.

\section{Introducción}

En la actualidad, la economía ha evolucionado hacia el desarrollo intensivo del sector servicios, desde ser considerado como un aspecto de la función mercadeo hasta el predominio de este tipo de empresas, donde en la mayoría de los casos el producto es un intangible. Por supuesto, este importante auge del sector servicios ha originado que se comiencen a estudiar las particularidades para su gestión.

La industria de los servicios ha crecido sostenidamente a nivel mundial en los últimos años, en términos de aportes al producto interno bruto y en la generación de empleos. Sin embargo, son escasas las investigaciones referidas a la gestión de las empresas de servicios, particularmente la gestión de la innovación en este sector.

Por su parte, el estudio y análisis de la innovación comienza a partir de la segunda mitad del siglo $\mathrm{XX}$, investigadores del área económica comienzan a profundizar en la importancia de la variable tecnológica en la creación de riqueza y valor en las empresas. Las primeras investigaciones se dirigieron hacia el problema de cómo medir los resultados de las inversiones realizadas en las actividades científicas y tecnológicas. Es así como la Organización para la Cooperación y Desarrollo Económico, OCDE, genera la primera versión de una herramienta para medir los resultados de las actividades científicas y tecnológicas en las empresas y en los países, denominadas Manual de Frascati (1980), cuya versión actual es el Manual de Oslo (1997). Esta herramienta se fundamenta en medir las entradas y salidas del sistema científico/tecnológico, y poco se ocupa en evaluar lo que ocurre en la caja negra de dicho sistema. Existe una amplia gama de metodologías para medir las actividades científicas/tecnológicas/innovación; sin embargo, en su mayoría están dirigidas a medir estas actividades en empresas de manufactura, tales como el Manual de Bogotá, el Cuadro Europeo de Innovación, entre otros.

Muchas empresas grandes instalaron sus unidades de Investigación y Desarrollo, I+D, donde se invertían cuantiosos recursos. Sin embargo, las estrategias y crecimiento de estas empresas no eran proporcionales al nivel de los recur- 
sos invertidos. A principio de los años 80 's, se comienza a plantear que no es suficiente la investigación y el desarrollo tecnológico, si este no incorpora el carácter económico que debe complementar esta actividad, entonces aparece la innovación como la principal herramienta para generar ventajas competitivas en las empresas.

En este contexto, se plantea como objetivo de este trabajo desarrollar un modelo conceptual para la gestión y evaluación de la innovación en empresas del sector servicios. La metodología utilizada para desarrollar este trabajo fue en primera instancia una revisión exhaustiva de los modelos existentes para medir la innovación, la calidad de servicios y la excelencia empresarial. Luego se generó el modelo conceptual propuesto, el cual fue revisado por expertos y actualmente está siendo validado empíricamente en diferentes subsectores de la industria de servicios.

El desarrollo de este artículo se lleva a cabo en tres etapas, en la primera se revisan las bases conceptuales que caracterizan a las empresas del sector servicios. En la segunda etapa se analizan las metodologías existentes para gestionar y medir la función de innovación y los modelos de gestión empresarial. En la tercera etapa se desarrolla el modelo propuesto para gestionar la innovación en el sector servicios.

\section{Características del Sector Servicios}

A nivel mundial se evidencia en cuanto a beneficios y empleos generados, que en las tres últimas décadas es el sector servicios o la industria de servicios quien ha tenido un crecimiento sostenido, a diferencia del sector de manufactura. La noción de servicios nace como una actividad propia del área de la mercadotecnia, donde la empresa debía garantizar un servicio postventa de sus productos para garantizar la satisfacción de sus clientes; en la medida que este servicio se especializaba, la empresa se diferenciaba de sus competidores y obtenía mayor fidelidad de sus clientes.

En función de la evolución de las necesidades de la sociedad moderna aparecen nuevas tecnologías, surgen actividades económicas que antes no existían, como el comercio global, las telecomunicaciones, el turismo, servicio aéreo, comida rápida, servicios bancarios, etc. Es entonces, cuando se comienza a diferenciar el sector servicios de la manufactura, dadas sus particulares características.

Según (Lovelock et al., 2004: 4), un servicio "es un acto o desempeño que ofrece una parte a otra. Aunque el proceso puede estar vinculado a un producto físico, el desempeño es en esencia intangible y, por lo general, no da como resultado la propiedad de ninguno de los factores de producción". Entre las características más resaltantes de la industria de los servicios están:

- Naturaleza intangible

- Intervención directa del cliente en el proceso de producción

- La calidad se expresa en términos de la percepción del cliente

- No existe inventarios luego de ejecutado el servicio

- El tiempo de prestación del servicio es una variable clave

Según Puente (2005:14), "una de las características esenciales de los servicios es 
que el consumo consiste en un proceso; es decir, son ofrecidos mediante un procedimiento en el cual interviene el consumidor". Debido al grado de intervención del cliente en el proceso, existen factores que definen la calidad en la prestación del servicio, como es el caso del recurso humano, específicamente sus competencias y nivel de motivación para ejecutar el proceso, las cuales son percibidas directamente por el cliente; una falla humana puede ocasionar la pérdida de clientes por insatisfacción. En algunas empresas de servicios es la tecnología quien juega un rol fundamental, tal es el caso del sector financiero donde la tecnología de la información y las telecomunicaciones determinan la eficiencia en la ejecución de los procesos internos y en la interfaz con el cliente; llegando inclusive a cambios radicales en los métodos de trabajo internos de los bancos y en el diseño de los servicios financieros ofrecidos.

"La calidad del servicio se define en términos de la percepción del servicio que tiene el cliente versus sus expectativas" (Parasuraman et al., 1988: 21). Conocer las percepciones, expectativas y necesidades se convierte en la información fundamental para diseñar el proceso de producción del servicio. Finalmente, citar el aspecto del diseño del proceso de entrega del servicio, el cual puede ser físico o electrónico, dependiendo si el cliente está presente en el lugar donde se desarrolla el proceso de producción del servicio o puede acceder a éste vía electrónica. Los factores citados anteriormente definen y caracterizan el estilo gerencial que las empresas de servicios deben atender prioritariamente.
Parasuraman et al. (1988: 21), en su definición de calidad de servicio, "plantea 5 dimensiones que el cliente aprecia en el momento de recibir el servicio, como son tangibilidad, confiabilidad, capacidad de respuesta, garantía y empatía". La tangibilidad se refiere a la capacidad de infraestructura adecuada para prestar el servicio por parte del productor. La confiabilidad representa la capacidad para que el servicio se ejecute bajo los parámetros y tiempo previstos. Capacidad de respuesta es la voluntad de ayudar al cliente con una respuesta rápida. Garantía incluye competencia para prestar el servicio, voluntad, cortesía y comunicación, es decir la actitud para prestar el servicio. Empatía son todos los esfuerzos dirigidos a comprender las necesidades del cliente, entender al cliente.

Muchos de los conceptos de gestión de empresas de manufactura pueden ser aplicables al sector servicios, realizando las adaptaciones correspondientes. Por ejemplo, para que una empresa de servicios funcione con criterios de excelencia se debe cultivar en todos los ámbitos de la organización una cultura de servicios, lo cual implica: a) Definir el Servicio a prestar, b) Definir el segmento del mercado que se va atender, c) Diseñar la máquina del servicio; es decir diseñar el proceso de producción del servicio y d) Definir un sistema de información que incluya los indicadores clave que sustentaran el negocio de servicios.

Otro aspecto importante en la industria de servicios, son los diversos criterios utilizados para su clasificación, tales como el grado de contacto con el cliente, intangibilidad, por tipo de industria, siendo este último uno de los más uti- 
lizados principalmente para generar estadísticas del sector. En Venezuela el Banco Central BCV, clasifica las actividades del sector servicios en: Comercio, Restaurantes y Hoteles, Transporte y Almacenamiento, Instituciones Financieras y Seguros, Bienes Inmuebles, Servicios Prestados a las Empresas, Servicios Comunales y Productores de Servicios. En 2004, el nivel de actividad del sector servicios representó el $60 \%$ de la actividad no petrolera, según BCV (2005); y en 2005 creció alrededor un $16 \%$ con predominio de los sectores comercio y telecomunicaciones. Estas cifras son equivalentes en otros países independientemente de su nivel de desarrollo, donde según la OCDE éste sector aporta por encima del $50 \%$ del PIB y genera la mayor cantidad de empleo.

En virtud de las particularidades que caracterizan al sector servicios es necesario adaptar las técnicas y herramientas para su gestión y administración. En este artículo se profundiza en como gestionar la innovación, factor determinante en la competitividad del negocio de servicios.

\section{Enfoques para gestionar la innovación y modelos de gestión empresarial}

\subsection{Metodologías para la gestión de la innovación}

Antes de revisar los diferentes enfoques para gestionar la innovación, es importante definir algunos conceptos útiles para sustentar el objetivo de esta investigación. "Por innovación se entiende todo desarrollo tecnológico apli- cado y con aceptación en el mercado", Arzola (2001:2). Existen diferentes tipologías de innovación, las tecnológicas que se sustentan en nuevos conocimientos científicos y/o tecnológicas, algo totalmente nuevo, generalmente están dirigidas a los productos y procesos de producción. Las organizativas, referidas a las mejoras o nuevas herramientas utilizadas en los procesos organizativos de las empresas. Y las innovaciones presentacionales que se refieren a la actividad de innovación en la función de mercadotecnia de la empresa. En el caso de las empresas del sector servicios, las innovaciones más frecuentes son las organizativas, según estudios empíricos de GaIlouj (1998) y Miles (1994).

La tecnología, está definida como el conjunto de conocimientos, métodos y herramientas utilizadas para producir bienes y servicios. Se puede hablar entonces de tecnología dura, referida al componente tangible de la tecnología, y tecnología blanda cuando se refiere a los conocimientos, aspecto intangible de la tecnología (Hidalgo et al., 2002). Otro concepto importante para el desarrollo de este estudio es el de actividades científicas, definidas como todo el proceso para generar nuevo conocimiento en las ciencias básicas, denominado conocimiento básico, el cual es aplicado para resolver cualquier problema que se convierte en conocimiento aplicado, también llamado tecnología. Para que ocurra innovación, es necesario que se generen, en la cantidad necesaria y suficiente, tanto el conocimiento básico como el aplicado.

Compatibilizar la gestión de la innovación con la estrategia global de la empresa puede representar un arma 
estratégica difícil de imitar por sus competidores. Chica et al. (2005) proponen un modelo conceptual para vincular la estrategia competitiva de la empresa con la correcta aplicación de las relaciones entre competitividad-estrategia-innovación-gestión.

Kline (1985) y la Asociación Española de Normalización y Certificación, AENOR (2006), proponen un modelo matricial, no lineal y concurrente para describir el proceso de innovación en las organizaciones, ver Diagrama 1. Esta herramienta permite conocer cada etapa de la innovación, así como también todos los parámetros y actores involucrados en cada una de sus fases. En este modelo se concibe la innovación como un proceso que comprende desde la generación de una idea hasta su explotación comercial, aplicable en su totalidad a empresas del sector servicios. Involucra por lo tanto aspectos tangibles e intangibles muy complejos de dirigir; razón por la cual son muchas las investigaciones realizadas sobre la gestión eficaz del proceso de innovación. Es importante destacar el aspecto de la retroalimentación, que sus fases ocurren concurrentemente e intervienen diferentes áreas funcionales tales como mercadeo, ingeniería, I+D, producción, comercialización, entre otras.

Según Betz (1998) la complejidad en la gestión de la innovación en las empresas viene dada por los diversos sistemas que constantemente están interac-

\section{Diagrama 1. \\ Modelo cadena/eslabón del proceso de innovación tecnológica}

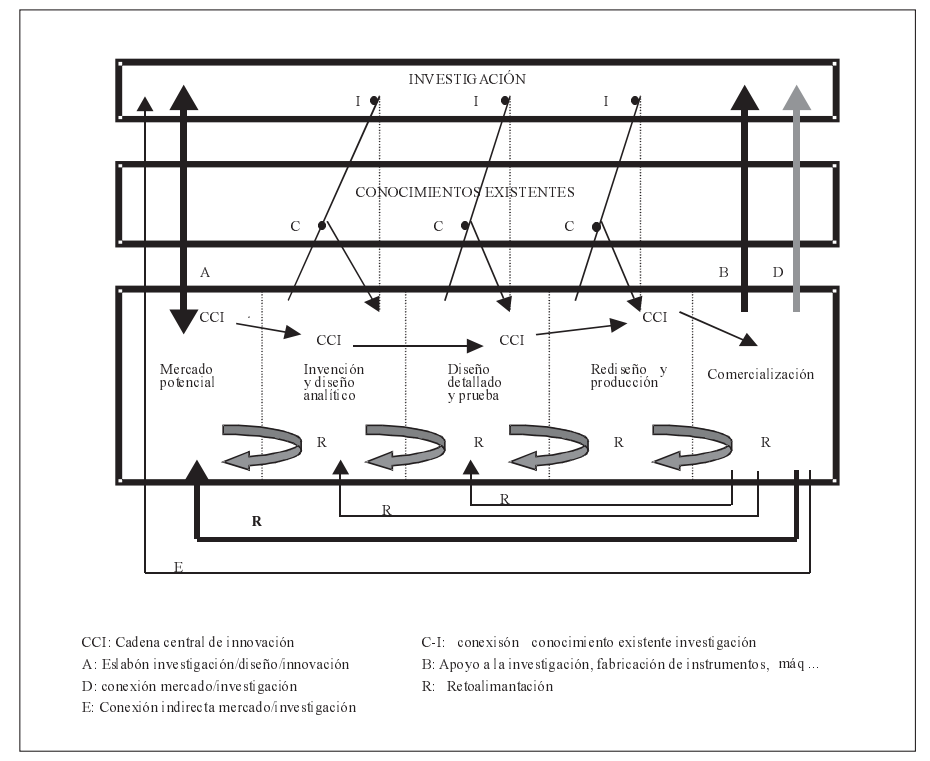

Fuente: Kline (1985). 
tuando en el proceso de innovación, lo que da origen a un cambio permanente. Deben combinarse algunas técnicas para una gestión exitosa del proceso de innovación, tales como análisis organizacional y de sistemas, planificación y predicción de la tecnología, gerencia de proyectos, marketing y técnicas para emprender nuevos negocios.

Pavón e Hidalgo (1997:65), definen la gestión de la innovación tecnológica como "el proceso orientado a organizar y dirigir los recursos disponibles, tanto humanos como técnicos y económicos, con el objetivo de aumentar la creación de nuevos conocimientos, generar ideas que permitan obtener nuevos productos, procesos y servicios o mejorar los existentes, y transferir esas mismas ideas a las fases de fabricación y comercialización".

Como proceso empresarial la innovación tiene seis funciones fundamentales, Morin (1985) y Escorsa y Valls (2003), cuyo cumplimiento conduce a su gestión eficaz, a saber:

1. Inventariar. Consiste en identificar las tecnologías internas esenciales en la actividad de la empresa. Se orienta a obtener información del estado actual de esas tecnologías, en el caso del inventario interno se denomina inventario tecnológico y debe clasificar las tecnologías en emergentes, claves y básicas en función del rol que desempeñen en la estrategia competitiva. Algunas herramientas útiles para realizar este inventario son la matriz tecnología/producto, el árbol tecnológico y el contenido tecnológico en cada etapa de la cadena de valor de la empresa.

2. Vigilar: Proporciona información sobre la aparición y evolución de nuevas tec- nologías y estima su posible impacto sobre las actividades de la empresa, las oportunidades y amenazas tecnológicas, la dinámica de las nuevas tecnologías y la probable secuencia de aplicación temprana, así como las barreras críticas al desarrollo de las mismas y las acciones futuras de los competidores. Para ejecutar esta función, la empresa debe mantener un sistema de alerta o vigilancia tecnológica, con fuentes de información fiables, dirigida por expertos multidisciplinares, con muy buenas relaciones con la comunidad técnica externa, a objeto de lograr un seguimiento efectivo de la evolución tecnológica externa y evaluar continuamente las posibilidades de adaptar las nuevas tecnologías a la empresa.

3. Evaluar: Luego de inventariar los recursos tecnológicos y establecer los mecanismos de vigilancia tecnológica, el paso siguiente es evaluar el nivel tecnológico y el potencial tecnológico de la empresa. Para estimar el potencial tecnológico, es de gran utilidad la matriz atractivo tecnológico/posición tecnológica, la cual combina una serie de variables para posicionar tecnológicamente la empresa. El atractivo tecnológico lo integran variables tales como potencial para generar nuevos productos, crecimiento del mercado, reducción de costos, mejoras en la calidad y cantidad de competidores que usan dicha tecnología. La posición tecnológica describe el dominio logrado por la empresa sobre cada tecnología, valorando variables como el liderazgo en la introducción de la tecnología, los gastos en actividades de I+D+l, la 
competencia del equipo investigador, el número de patentes o la red de relaciones.

4. Enriquecer: En función de la estrategia tecnológica resultante de la fase anterior, esta función plantea incrementar el patrimonio tecnológico de la empresa. Consiste en identificar las diferentes opciones para adquirir las tecnología requeridas, ya sea por desarrollo interno de actividades de I+D o en el exterior mediante adquisiciones de empresas que posean dicha tecnología, licencias, proyectos conjuntos o alianzas estratégicas 0 proyectos de colaboración con universidades o centros públicos de investigación.

5. Optimizar: Consiste en buscar la mejor utilización posible de los recursos tecnológicos; es decir, la explotación del potencial tecnológico mediante el análisis sistemático de nuevas aplicaciones en otras áreas que brinden oportunidades para obtener beneficios.

6. Protección. Existen diferentes opciones legales que le permiten a la empresa proteger sus innovaciones tecnológicas, denominadas propiedad industrial, las cuales otorgan un derecho exclusivo de explotación durante un período de tiempo. Aunque cada país tiene sus leyes, de manera genérica las formas de propiedad industrial se agrupan en cuatro modalidades:

- Protección de invenciones: los avances tecnológicos producidos se protegen a través de las patentes y los modelos de utilidad, consistente en un derecho de carácter monopolista de uso exclusivo de una invención.
- Protección de marcas: la marca es el símbolo distintivo de la empresa, de sus productos y servicios, si se usa puede tener carácter indefinido.

- Protección del diseño industrial: el diseño industrial es una de las etapas más creativas del proceso de innovación tecnológica, puede protegerse mediante los modelos y dibujos industriales que estén dirigidos a la protección de los rasgos ornamentales, de estructura y configuración externa.

- Protección del saber hacer: también conocido como know how, es el conjunto de conocimientos relacionados con el proceso de desarrollo tecnológico, que deliberadamente se mantiene confidencial, dura mientras se mantiene en secreto. Su protección se realiza bajo la figura de licencias de conocimiento.

Markides (1997) es partidario que las empresas incorporen la cultura de innovación en su funcionamiento cotidiano y propone una rutina para iniciar este proceso de incorporación:

- Redefinir el negocio.

- Redefinir el quién: ¿quiénes son nuestros clientes?, pensar en nuevos clientes o nuevos segmentos y desarrollar planes para atenderlos mejor.

- Redefinir el qué: ¿qué productos o servicios ofrecemos a esos clientes?, pensar en las nuevas necesidades o deseos de los clientes y desarrollar un plan que los satisfaga mejor.

- Redefinir el cómo: aprovechar las competencias claves para desarrollar nuevos productos y mejorar el negocio para luego encontrar los clientes adecuados. 
- Iniciar el proceso mental en diferentes momentos en el tiempo: ¿cuáles son las capacidades singulares?, ¿qué necesidades específicas podemos satisfacer?, ¿quiénes serán los clientes adecuados que se deben atender?

En el 2002, AENOR propuso una serie UNE 166000 EX, Normas Españolas experimentales referidas a la Gestión de la I+D+l. Esta serie la conforman un conjunto de 5 normas, específicamente la norma 166002 denominada Requisitos del Sistema de Gestión de la I+D+l, es la que se refiere a la gestión de la innovación y cuyo esquema de desarrollo es el de las normas ISO 9000: 2000. En dicha norma es una descripción detallada de todas las actividades de I+D+I que las empresas deben cumplir para garantizar que se gestione adecuadamente la innovación, a saber: las herramientas para la I+D+l, identificación y análisis de problemas y oportunidades, análisis y selección de ideas de I+D+I, planificación, seguimiento y control de la cartera de proyectos, transferencia de tecnología, producto de la I+D+l, compras, resultados del proceso de I+D+l y protección y explotación de los resultados de las actividades de $\mathrm{I}+\mathrm{D}+\mathrm{I}$.

Delgado et al. (2002), proponen un modelo integrado para gestionar la innovación, el cual incluye la gestión estratégica, la de recursos humanos, la de la calidad y la dirección integrada de proyectos. Otros investigadores como Torres et al. (1986), Santarelli y Piergiovanni (1996), Arzola (2001) han realizado interesantes aportes teóricos de cómo medir el proceso de innovación, particularmente en las empresas.
Todas las metodologías descritas anteriormente están orientadas a medir el proceso innovador en empresas manufactureras, para aplicarlas a las empresas del sector servicios es necesario adaptarlas.

Según Bilderbeek et al. (1988: 26), afirman que "la actividad innovadora en el sector servicios se puede describir en cuatro dimensiones: concepto de nuevo servicio, nueva interfaz con el cliente, nuevos sistemas de distribución y entrega, y las opciones tecnológicas, y para que suceda la innovación implica una combinación de dichas dimensiones". La Dimensión 1 se refiere al concepto de nuevo servicio, es decir la innovación sucede cuando se ofrecen servicios nuevos a los clientes. La dimensión 2 puede suceder cuando la empresa asume nuevas formas de interfaz con el cliente, en muchos casos apoyadas en las tecnologías de la información y las comunicaciones, adaptadas a las necesidades cambiantes de los clientes. La dimensión 3 nuevos sistemas de distribución y entrega del servicio, se refiere a los arreglos organizativos internos que se hacen para permitir a los trabajadores realizar mejor su trabajo y ofrecer los productos adecuadamente. La dimensión 4 , se refiere a las opciones tecnológicas, cada tipología de servicio requiere en diferentes niveles de la tecnología para proveer sus servicios; por tanto es necesario conocer las opciones tecnológicas disponibles.

Este modelo de innovación en el sector servicios, caracteriza minuciosamente los tipos de innovación que pueden ocurrir en este tipo de organizaciones, tales como los nuevos servicios y los nuevos sistemas de distribución; sin em- 
bargo, no incluye dimensiones referidas a la gestión de la innovación, la cual garantizarían el desarrollo y consolidación de las organizaciones de servicios.

\subsection{Modelos de Gestión Empresarial}

Producto de la dura y compleja competitividad existente entre las empresas en casi todos los sectores económicos a nivel global, en los últimos años, algunos países se han preocupado por promocionar la calidad, competitividad y excelencia en sus empresas creando algunos modelos para premiar las mejores prácticas en las empresas, Cuadro 1. Todos concebidos como instrumentos de evaluación/comparación con el deber ser de las organizaciones para responder a un entorno de alta complejidad y competencia. Estos seis modelos tienen como objetivo promover la calidad y la excelencia de gestión en las organizaciones. Del análisis de los aspectos conceptuales de cada uno de ellos, se aprecia que existen dimensiones comunes a la mayoría de los modelos, como son los aspectos gerenciales del liderazgo, la planificación estratégica, los recursos humanos, los clientes y la medición de resultados. Las diferencias más significativas entre estos seis modelos de calidad y excelencia son el enfoque de procesos de los modelos más recientes y el despliegue de tales dimensiones en el proceso de implementación. Es así como estas dimensiones constituyen la base teórica para generar el modelo conceptual para gestionar la innovación en las empresas del sector servicios.

La primera experiencia en materia de este tipo de modelo fue la de Japón, que en 1951 comenzó a otorgar el Premio
Deming a las empresas que realizaran esfuerzos exitosos para implantar principios corporativos de control de calidad. Este premio lo lidera la Unión Japonesa de Científicos e Ingenieros (JUSE), su enfoque principal es el control estadístico de la calidad, evalúa aspectos como: políticas y objetivos, organización y operación, educación y extensión, recopilación de datos, normalización y aseguramiento de la calidad (JUSE, 2004).

El Premio Nacional de Calidad Malcolm Baldrige, tiene por objetivo estimular y apoyar la mayor competitividad de Estados Unidos a través de un reconocimiento del gobierno a la calidad excepcional en las empresas del país. El mismo consiste en evaluar las organizaciones en función de un sistema de gerencia de calidad total y las mejoras que se generan, basado en la solidez del enfoque, la integración y los resultados de dicho sistema, NIST (2005). Se evalúan siete aspectos que definen la calidad y la excelencia de gestión, a saber: liderazgo, información y análisis, planificación estratégica de la calidad, utilización de recursos humanos, aseguramiento de la calidad de productos y servicios, resultados de calidad y satisfacción de los clientes. Para la evaluación de los sietes aspectos anteriores se asigna un máximo de 1000 puntos, repartidos de acuerdo al impacto del aspecto en los resultados de la empresa.

El Premio Europeo a la Excelencia, auspiciado desde 1991 por la Fundación Europea para la Gestión de la Calidad, EFQM, es un mecanismo de estimulo para que las empresas adopten la filosofía de excelencia de gestión basado en la innovación y el aprendizaje organizacional, considera la evaluación de los agentes facilita- 


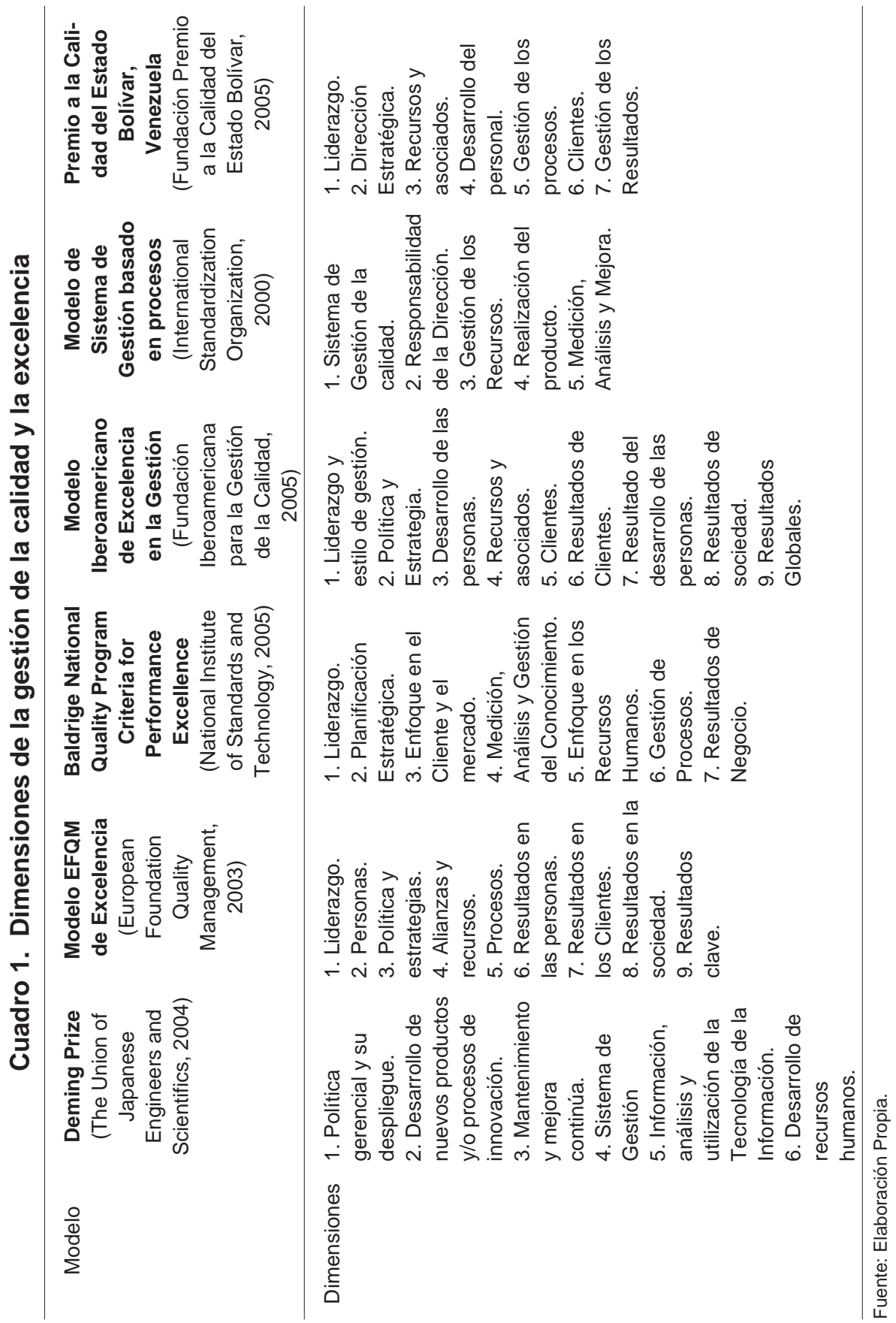


dores y los resultados de la excelencia (EFQM, 2003). Como instrumento de evaluación introduce conceptos interesantes en la gestión de empresas, como son el enfoque de procesos, las alianzas como mecanismos para crear ventajas competitivas y los resultados para los diferentes grupos de interés en la empresa. Los aspectos evaluados en este premio son, liderazgo, política y estrategia, personas, alianzas y recursos, procesos, resultados en clientes, resultados en personas, resultados en la sociedad y resultados claves. Asigna un total de cien puntos para la evaluación total.

El Modelo Iberoamericano de Excelencia en la Gestión, se fundamenta en promocionar la innovación y la mejora continua de las empresas Iberoamericanas. Este premio es auspiciado por la Fundación Iberoamericana para la Gestión de la Calidad desde el año 2000. Su estructura de evaluación es similar a la del Premio Europeo, su base de evaluación son 1000 puntos.

El Premio a la Calidad del Estado Bolívar/Venezuela, es la primera experiencia de este tipo en el país, se inicia en el año 2003 como un reconocimiento a la calidad en las empresas. Actualmente, instituciones regionales públicas y privadas interesadas en promover la excelencia y la calidad en las empresas consolidaron la Fundación del Premio a la Calidad del Estado Bolívar, quien desde el 2004 otorga este premio a las organizaciones del Estado Bolívar.

\subsection{Modelos de Calidad de Servicios}

El método SERVQUAL, Parasuraman et al. (1988:13), propone que la "cali- dad de servicios puede medirse en función de la satisfacción del cliente en términos de comparar la percepción del servicio recibido con sus expectativas iniciales. Cuando las expectativas son superadas, el servicio se puede denominar de excepcional calidad; cuando no se cumplen las expectativas del cliente, el servicio se califica como inaceptable; cuando se cumple exactamente el nivel de expectativas del cliente se define como servicio de calidad satisfactoria". Para medir el nivel de calidad de servicios, estos autores proponen el modelo de escala múltiple SERVQUAL, el cual mide cinco dimensiones (tangibilidad, confiabilidad, capacidad de respuesta, garantía y empatía) que incluyen una serie de variables asociadas a calidad de servicios.

Wels-Lips et al. (1998:288), plantean que medir los incidentes críticos positivos y negativos en el momento de la verdad; es decir, cuando el cliente está recibiendo el servicio; cómo suceden estos incidentes críticos definen la calidad de servicios para el cliente. Las dimensiones del servicio se dan en función de la tipología del servicio.

\section{Modelo Conceptual para la Gestión de la Innovación en el Sector Servicios, 7D's}

La metodología utilizada para desarrollar el Modelo Conceptual para la Gestión de la Innovación en el sector servicios, 7D's, fue en primer lugar la evaluación y análisis de las bases teóricas relacionadas con el tema; cuyo hallazgo más relevante fue la escasa literatura al respecto. En segundo lugar, se analizaron los modelos de gestión de la innovación, 
los cuales en su mayoría se dedican al sector de manufactura. En este sentido, se combinaron los factores indispensables para garantizar el éxito de los negocios con la inclusión de factores fundamentales en la prestación de cualquier servicio.

El modelo que a continuación se propone responde a la necesidad de contar con un modelo de gestión de la innovación en las empresas del sector servicios cuya filosofía sea la gestión de excelencia en el sector servicios. Este modelo incluye los aspectos referidos a la gestión integral del negocio, el tipo de innovación organizativa, la más frecuente en la industria de los servicios, los procesos, el recurso humano y la satisfacción del cliente como elemento de que define la calidad del servicio. Es decir, este modelo se fundamenta en los criterios establecidos en los modelos de excelencia de gestión, los de medición de la innovación y en la principal característica de la industria de servicios como es el nivel de satisfacción de los clientes; de estas bases conceptuales se derivan las siete dimensiones que conforman el modelo 7D's.

Comparando el modelo 7D's con el propuesto por Bilderbeek (1998), la diferencia fundamental entre ambos, es que el modelo propuesto en este artículo es una herramienta de evaluación de la gestión de la innovación; mientras Bilderbeek plantea una descripción de los tipos de innovación que pueden ocurrir en las empresas de servicios.

En otro orden de ideas, la principal diferencia entre los modelos que miden la calidad del servicio, y el modelo propuesto, es que mientras los primeros miden la calidad de servicio valorando la percep- ción de los clientes externos; el modelo 7D's y su instrumento de evaluación están diseñados para ser aplicados en las áreas funcionales de la organización, es decir constituye una herramienta de evaluación interna para las empresas.

El modelo 7D's mide siete dimensiones necesarias para la gestión de la función de innovación en las empresas en el sector servicios, a saber: Liderazgo, Planificación Estratégica, Competencias del Recurso Humano, Procesos, Organización, Satisfacción de Clientes y Responsabilidad Social (ver Diagrama 2). Cada una de estas siete dimensiones está compuesta por una serie de variables que caracterizan dicha dimensión.

Este modelo propone que cada una de las siete dimensiones ocurran de manera concurrente, interrelacionadas y atendiendo cada una de las variables consideradas en cada dimensión. Es decir, las siete dimensiones propuestas por el modelo 7D's deben interactuar simultáneamente para alcanzar el nivel de innovación y la calidad del servicio esperada por los clientes. Por lo tanto, los directivos de las empresas de servicios deben esforzarse para que cada una de estas dimensiones tenga un grado de desempeño óptimo, a objeto que se puedan superar las expectativas del cliente.

Las dimensiones Liderazgo y Planificación Estratégica presentadas en la parte superior del diagrama del modelo e interactuando entre ellas, evalúan como se gerencia el negocio de servicio y si la empresa incluye aspectos de innovación en sus prácticas gerenciales. Las dimensiones Procesos, Competencias del Recurso Humano y Organización, ubicadas en el eje central del modelo representan 


\section{Diagrama 2 \\ Modelo para medir la innovación en el sector servicios}

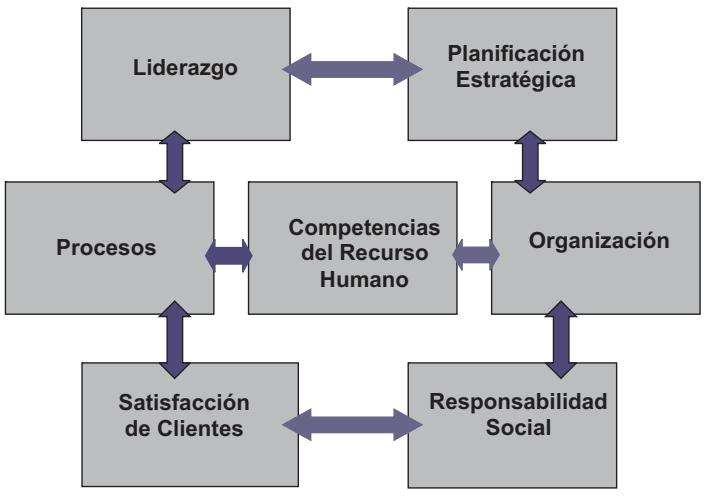

Fuente: Elaboración Propia.

la máquina para la prestación del servicio y se refieren a la infraestructura física y la intangible necesaria para ejecutar el servicio, como están organizados los procesos y el rol del recurso humano como prestador del servicio. Finalmente, las dimensiones Satisfacción de Clientes y Responsabilidad Social ubicadas en la parte inferior del modelo, como elementos de valoración de los resultados del negocio de servicio. Es importante destacar, que la dimensión Competencias del Recurso Humano se ubica en el centro del modelo, como motor del mismo, dada la importancia que tiene en la ejecución de la función de innovación en las empresas de servicio.

La dimensión Satisfacción de Clientes, se refiere al grado de satisfacción del cliente y agrupa un conjunto de variables vinculadas a la innovación del servicio y a la percepción que tiene el cliente una vez recibida la prestación del servicio. Las variables consideradas son: tipos de servicios, frecuencia en la incorporación de nuevos servicios, percepción del cliente, satisfacción, necesidades, innovación, calidad de servicios, quejas y reclamos, interfaz, entre otras.

Competencias del Recurso Humano se refiere a los conocimientos, habilidades y formación del personal necesarios para que el servicio ocurra en la calidad y oportunidad esperada por el cliente. Las variables analizadas son, formación, capacitación, participación en programas de mejoras e innovación, creatividad, trabajo en equipo, motivación, recompensas, sistema de gestión del $\mathrm{RRHH}$ y las fuentes de aprendizaje tecnológico.

La dimensión Procesos se refiere a la medición de los aspectos asociados con la infraestructura de los procesos necesarios para la prestación del servicio. Es importante destacar, que esta infraestructura de los procesos puede tener carácter tangible o netamente intangible. Su 
importancia radica en que en el sector servicios en muchos casos, el cliente debe interactuar directamente en el proceso, razón por la cual de su diseño y desempeño depende el éxito y la calidad del servicio prestado. Entre las variables evaluadas en esta dimensión están, tecnología utilizada, documentación, certificación, mejora continua, tipo, uso de la capacidad, entre otras.

Se incluye la dimensión Organización en este modelo, dado el predominio de la innovación de tipo organizativa en lugar de las innovaciones tecnológicas en el sector servicios. Es decir, las mejoras de los procesos y de las metodologías que soportan las actividades de las empresas de servicios son más frecuentes que los cambios radicales a nivel tecnológicos. Las variables asociadas a la dimensión Organización son, estructura organizativa, manuales, normas, procedimientos, sistemas de información, seguimiento y control, ambiente de trabajo adecuado y la incorporación de mejoras en estos ítems en los últimos años.

El Liderazgo se refiere a la importancia de ejercer liderazgo adecuado para poder ejecutar acciones de innovadoras en el sector servicios. Según Granell (2005:28) el liderazgo compartido en toda la organización, es esencial para convertir la estrategia en resultados. Las variables consideradas en esta dimensión son las siguientes, ejercicio de autoridad, responsabilidades, motivación, toma de decisiones, mecanismos para fomentar la innovación y políticas.

El uso del enfoque de Planificación Estratégica es una de las herramientas gerenciales que garantizan el crecimiento y competitividad de las empresas, las del sector servicio no escapan a este comportamiento, validado empíricamente en múltiples estudios, Frances (2004). La Planificación Estratégica dirige el desarrollo de las empresas enfatizando en el conocimiento y uso adecuado de las capacidades internas, considerando las oportunidades y amenazas del entorno para formular las estrategias a seguir. En esta dimensión se incluyen variables relacionadas con la innovación como elemento indispensable en la formulación de la visión, misión, estrategias, mejoramiento continuo, recursos invertidos en innovación, activos de propiedad intelectual, inversión en formación de personal, vigilancia, plan de desarrollo tecnológico.

La Responsabilidad Social es necesaria para cumplir con todos los grupos de interés de las empresas dadas exigencias actuales de la sociedad, clientes, accionistas, trabajadores, ambiente y la sociedad en general. Las variables que caracterizan la dimensión Responsabilidad Social, son, crecimiento en rentabilidad, continuamente ofrece nuevos productos y servicios, acciones para preservar el ambiente y acciones sociales que beneficien a la comunidad.

\section{Criterios de Evaluación}

En el modelo 7D's para evaluar la gestión de la innovación en el sector servicios, se ofrecen dos alternativas para evaluar cada variable del modelo, una escala del 1 al 5 o una valoración equivalente en términos del \% de cumplimiento, ver Cuadro 2, en el cual se describen tres columnas definidas como evaluación, \% de cumplimiento y la interpretación de cada valoración. La primera alternativa de evaluación cualitativa, propone un rango de valoración del 1 al 5 , donde la asignación 


\section{Cuadro 2 \\ Criterios para la evaluación de las dimensiones que caracterizan la innovación en el modelo 7D's}

\begin{tabular}{|c|c|c|}
\hline Evaluación & $\%$ de cumplimiento & Interpretación \\
\hline 1 & 0 & $\begin{array}{l}\text { Inexistente, no cumple ninguno de los elementos o requi- } \\
\text { sitos; o existe pero no se aplica. }\end{array}$ \\
\hline 2 & 25 & $\begin{array}{l}\text { Existe con debilidad, se aplica en algunas áreas, o par- } \\
\text { cialmente en todas las áreas. }\end{array}$ \\
\hline 3 & 50 & $\begin{array}{l}\text { Existe y se aplica en casi todas las áreas y niveles, con ca- } \\
\text { rácter puntual, no existe medición y seguimiento. }\end{array}$ \\
\hline 4 & 75 & $\begin{array}{l}\text { Existe y se aplica en toda la organización, con carácter } \\
\text { permanente, medición y seguimiento sistemático en toda } \\
\text { la organización. }\end{array}$ \\
\hline 5 & 100 & $\begin{array}{l}\text { Existe y se aplica en toda la organización con carácter } \\
\text { permanente, medición y seguimiento sistemático. Existen } \\
\text { tendencias a mejora e innovar continuamente. }\end{array}$ \\
\hline No aplica & & $\begin{array}{l}\text { La variable o aspecto no aplica para la actividad económi- } \\
\text { ca que se desarrolla }\end{array}$ \\
\hline
\end{tabular}

Fuente: Elaboración Propia.

de uno (1) corresponde a la inexistencia del cumplimiento del requisito de la variable evaluada, en la empresa que se esta diagnosticando; y la calificación de cinco (5) en una variable significa que la empresa la ejecuta continuamente, existen factores de medición y acciones de mejora continua; la interpretación de los valores 2, 3 y 4 se muestran en el Cuadro 2. De forma análoga se puede expresar la evaluación de cada variable en función del grado de su cumplimiento en la organización, la cual se expresa porcentaje.

Discusión conceptual del modelo

Tal como se ha planteado, el modelo 7D's se fundamenta en los criterios establecidos en los modelos de excelencia de gestión, los de medición de la innovación y en uno de los principales motores para la toma de decisiones en la industria de servicios como es conocer el nivel de satisfacción de los clientes. Adicionalmente, en función de las características de la industria de servicios, se prevé la evaluación de tres aspectos fundamentales que definen el éxito del negocio de los servicios: la gestión, la máquina de servicio, los resultados en los grupos de interés de la empresa incluyendo sus clientes.

La dimensiones liderazgo y planificación estratégica emergen como elementos que garantizan la gestión del negocio de servicios. La máquina de servicio o la infraestructura para prestar el servicio, que incluyen las dimensiones procesos, la organización y el recurso humano constituyen la base estructural para la prestación del servicio. El último aspecto incluye las dimensiones que miden los resultados de la prestación del servicio, la satisfacción de clientes y la responsabili- 
dad social de las empresas con sus grupos de interés.

Según los resultados de la revisión del estado del arte relacionado con la innovación el sector servicios, se evidencia una escasez de literatura e investigaciones empíricas sobre este tema. De allí se deriva la importancia y pertinencia del modelo propuesto, el cual debe validarse en organizaciones de servicios pertenecientes a diferentes sectores de actividad, por ejemplo, turismo, educación, construcción, telecomunicaciones, financieros.

\section{Conclusiones}

- Existe escasa literatura e investigaciones relacionadas con la gestión de la innovación en el sector servicios; el establecimiento del estado del arte en este tema, le da pertinencia y vigencia al modelo propuesto, así mismo, permitió delinear las bases conceptúales que sustentan dicho modelo.

- El origen conceptual del modelo 7D's se enmarca en las características de las empresas del sector servicios, los modelos de excelencia empresarial, las herramientas para medir la innovación y la calidad de servicio.

- El modelo 7D's para evaluar la gestión de la innovación en las empresas del sector servicios, es una herramienta de evaluación interna para las empresas. Cada una de las siete dimensiones del modelo 7D's, se derivaron de las bases conceptuales de los modelos de excelencia de gestión, de calidad de servicios y los de gestión de la innovación; Considerando los criterios gerenciales básicos para las empresas de servicios como son la gerencia de los procesos, la infraestructura de los procesos y los resultados esperados por los clientes.

- El modelo 7D's propuesto para la gestión de la innovación en el sector servicios, lo conforman siete dimensiones: Liderazgo, Planificación Estratégica, Procesos, Organización, Satisfacción de Clientes, Competencias del Recurso Humano y Responsabilidad Social. Es decir, para evaluar la gestión la innovación en las empresas del sector servicios, se deben valorar estas siete dimensiones.

- Los modelos de innovación en servicios desarrollados hasta la fecha, solo abordan el tema de los tipos de innovación que pueden ocurrir en las empresas de servicios; El modelo 7D's se orienta hacia la evaluación de la gestión interna del negocio de servicio, planteando además criterios cuantitativos y cualitativos que permitan una mejor interpretación de los resultados de la evaluación.

- En las empresas de servicio, las siete dimensiones propuestas por el modelo 7D's deben interactuar simultáneamente para alcanzar la calidad del servicio esperado por los clientes. Por lo tanto, los directivos deben esforzarse para que cada una de estas dimensiones tenga un grado de desempeño óptimo, a objeto que se puedan superar las expectativas del cliente. 


\section{Referencias Bibliográficas}

Asociación Española de Normalización y Certificación AENOR (2006). Norma 166002. Gestión de la I+D+i: Requisitos del Sistema de Gestión de la I+D. Madrid. $24 p$.

Arzola, M. (2001). Comparación de la Capacidad Tecnológica del Sector Metal entre Aragón/España y Guayana/Venezuela mediante Categorías Universales: Perspectivas para la Cooperación Empresarial. Tesis Doctoral. Universidad de Zaragoza. España. Pp. 1-27.

Banco Central de Venezuela, BCV (2005). Informe Anual 2004. Caracas. www.bcv.gov.ve 20/06/05.

Betz, F. (1998). Managing Technological Innovation, Competitive Advantage from Change. John Wiley \& Sons, Inc. New York.

Bilderbeek, R., Den Hertog, P., Marklund, G. y Miles, I. (1998). "Services en Innovation: Knowledge Intensive Business Services (KIBS) as Co-producers of Innovation". En SI14S Project. SI14S Synthesis Papers No. 3. STEP Group.

Chica, Y.; Bastidas, P. y Vargas, A. (2005). Innovación Tecnológica como Herramienta para la Competitividad en la Industria. Revista Creando. Año 2. Nro. 4. Universidad Nacional de Colombia. Manizales.

Delgado, M.; Marconi, E. y Olivera, H. (2002). Análisis Comparativo de Instrumentos de Evaluación de la Innovación Tecnológica. Revista Ingeniería Industrial. Vol. XXIII Nro. 3. pp. 56-63. La Habana.

Delgado, M. y Díaz, F. (2002). Enfoques integrados de la Gestión de la Innovación Tecnológica. Revista Ingeniería Industrial Vol. XXII Nro. 4. pp 29-37.

Escorsa, P. y Valls, J. (2003). Tecnología e innovación en la empresa, Segunda edición, Ediciones UPC, Barcelona. $271 \mathrm{p}$.

European Foundation for Quality Management - EFQM (2003): Modelo EFQM de Excelencia, EFQM, Brucelas, Belgica, $85 p$.

Fitzsimmos, J. y Fitzsimmos, M. 2001. Service Management. Mc Graw Hill. Décimo segunda edición. New York. pp 268317.

Frances, A. (2004). Estrategia para gente de a pie. Revista Debates IESA Vol. IX. Nro. 4. pp 12-16.

Fundación Premio a la Calidad del Estado Bolivar (2005). Premio a la Calidad Estado Bolívar, Ciudad Guayana, Venezuela, 22p.

Fundación Iberoamericana para la Gestión de la Calidad (2005). Modelo Iberoamericano de Excelencia en la Gestión, Edición 2005, Madrid, España, 23 p.

Granell, E. (2005). Más allá de la satisfacción al cliente: ¿cómo convertir la estrategia en resultados. Revista Debates IESA Vol. X. No. 3. pp. 25-28.

Gallouj, F. (1998). Innovation in Reverve Services and the Reverse Product Cycle. En: SI4S Topical Paper, No 5. STEP Group.

Hidalgo, A.; León, G. y Pavón, J. (2002). La gestión de la Innovación y la tecnología en las organizaciones. Ediciones Pirámide. 559p.

International Standardization Organization ISO (2000). Norma Internacional ISO 9000: Sistemas de Gestión de la Calidad, Definiciones y vocabulario, Ginebra, Suiza, 29 p.

Kline, S. (1985). Innovation is not linear process. Research Management. JulioAgosto. pp. 36-45.

Lovelock, C.; Reynoso, J.; D’Andrea, G. y Huete, L. 2004. Administración de Servicios. Pearson-Prentice Hall. Mexico. pp 1-35. 
The Union of Japanese Engineers and Scientifics-JUSE (2004). The Guide for Deming Application Prize Guide 2006 for overseas. The Deming Prize Committee, Japan, $51 \mathrm{p}$.

Markides, K. (1997). La Innovación Estratégica. Harvard Deusto Businness Review. Nov/Dic Nro. 81. pp 29-40.

Miles, I. (1994). Innovation in Services. Part 2: Sectoral and Industrial Studies of Innovation en The HandbooK of Industrial Innovation. Editores M. Dodgson, M. y Rothwell, R. Pp. 243-256. Gran Bretana.

Morin, J. (1985). L'excellence technologique. Publi Union. Paris. 177p.

National Institute of Standards and Technology NIST (2005). Baldrige National Quality Program, 2006 Criteria for Performance Excellence, Gaithersburg. MD. USA. 81 p.

Organisation for Economic Co-operation and Development OECD (1997). Oslo Manual. Bruselas.

Organisation for Economic Co-operation and Development OECD (1980). Frascati Manual. Paris.

Parasuraman, V.; Zeithaml, A.; Berry, L. (1988). "SERVQUAL: A Multiple Item Scale for Measuring Costumer Perceptions of Service Quality". Journal of Retailing. Vol 64, Nro 1. Spring pp 1240
Pavón, J., e Hidalgo, J. (1997). Gestión e Innovación, un Enfoque Estratégico. Ediciones Pirámide. Madrid. pp 6384.

Puente, R. (2005). "Del mercadeo de servicios a la gerencia de servicios". Revista Debates IESA. Vol. X. Nro. 3. Caracas. pp. 13-16.

Santarelli, E. y Piergiovanni, R. (1996). "Analyzing literature-based innovation output indicators: the Italian experience". Research Policy. Vol. 25. pp 689-711.

Torres, F.; Martínez, A. y Poza, P. (1986). Inventario Tecnológico de Aragón. Diputación de Aragón. España. pp35-53.

Wels-Lips, I.; Van der Ven, M. y Pieters, R. (1998). Critical services dimensions: an empirical investigation across six industries. En revista International Journal of Service Industry Management. Vol. 9 Nro. 3.pp 286-309. MBC University Press. 